\title{
Alkylation studies of a polyhydroxylated-cyano-piperidine scaffold
}

\author{
Alicia M. Dilmaç, ${ }^{a}$ Tony Tite, ${ }^{\text {a Andriamihamina Tsimilaza, }{ }^{\text {a }} \text { Pascale Lemoine, }}$, \\ Sabrina Boutefnouchet, ${ }^{a}$ Sylvie Michel ${ }^{a}$ and Marie-Christine Lallemand ${ }^{{ }^{*}}$
}

${ }^{a}$ Laboratoire de Pharmacognosie, UMR CNRS 8638 Université Paris Descartes, Sorbonne Paris Cité, Faculté des Sciences Pharmaceutiques et Biologiques, 4, Avenue de l'Observatoire, 75006 Paris, France

${ }^{b}$ Laboratoire de Cristallographie et RMN Biologiques UMR CNRS 8015 Université Paris Descartes, Sorbonne Paris Cité Faculté des Sciences Pharmaceutiques et Biologiques, 4, Avenue de l'Observatoire, 75006 Paris, France

E-mail: marie-christine.lallemand@parisdescartes.fr

\section{DOI:http://dx.doi.org/10.3998/ark.5550190.0015.403}

\begin{abstract}
Hexahydro-3-phenyl-6,7,8-trihydroxy-3R-[3 $\alpha, 5 \beta, 6 \beta, 7 \alpha, 8 \beta, 8 \mathrm{a} \beta]-5 H$-oxazolo[3,2-a]pyridine-5carbonitrile is endowed with two non equivalent reactive sites: an $\alpha$-amino nitrile at the C-5 position and an $\alpha$-amino ether at the C-8a position. Herein, alkylation at the C-5 position was studied. The scope and limitations of these reactions have been investigated.
\end{abstract}

Keywords: Trihydroxy-cyano piperidine scaffold, alkylation studies, scope and limitations

\section{Introduction}

Polyhydroxylated piperidine and indolizidine alkaloids, called iminosugars, are important targets in organic synthesis due to their potential value as glycosidase inhibitors. ${ }^{1-4}$ These compounds have been designed as therapeutic solutions in several diseases ${ }^{5-10}$ (type II diabetes, viral and bacterial infections, lysosomal storage disorders, tumor metastasis). They have also been found in a number of natural ${ }^{11-16}$ or synthetic ${ }^{17-24}$ heterocyclic compounds, such as castanospermine and $N$-butyl-deoxynojirimycin ( $N$-butyl-DNJ) respectively (Figure 1$)$.

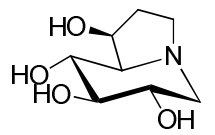

castanospermine

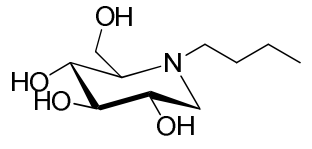

N-butyl-DNJ

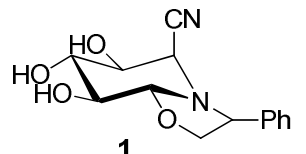

Hexahydro-3-phenyl-6,7,8-trihydroxy$3 R-[3 \alpha, 5 \beta, 6 \beta, 7 \alpha, 8 \beta, 8 \mathrm{a} \beta]-5 \mathrm{H}-$ oxazolo-[3,2-a]pyridine-5-carbonitrile

Figure 1 
Chemists have been inspired by the numerous pharmacological activities of iminosugars, but unfortunately some of them are also toxic to human cells. Nevertheless, there is still a need for an easy access to alkaloid analogs and for the development of more potent, selective and less toxic drug candidates. In this context, we decided to consider the chiral building block 1 (Figure 1) as starting material. ${ }^{25}$ As a part of our ongoing research program on the reactivity of compound 1, we already reported a rapid access to substituted six- or seven- membered ring iminosugars via ring-expansion reactions ${ }^{26}$ and a new asymmetric synthesis of $(2 S, 3 R, 4 R, 5 S)$ trihydroxypipecolic acid. ${ }^{27}$ In order to prepare indolizidine analogs such as castanospermine derivatives, we have extended, herein, our research on the alkylation study at the C-5 position of the building block 1 (Scheme 1). Compound 1 possesses two non-equivalent reactive sites on the polyhydroxylated piperidine ring system: an $\alpha$-amino nitrile at the $\mathrm{C}-5$ position and an $\alpha$-amino ether at the $\mathrm{C}-8$ a position.

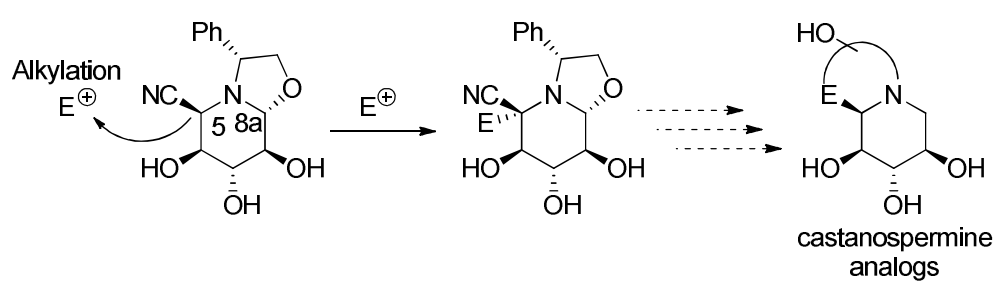

\section{Scheme 1}

\section{Results and Discussion}

In this paper, we focus on the methylation reactions at the $\mathrm{C}-5$ position. The scope and limitations of this alkylation are discussed. Our purpose is to compare the reactivity of our building block 1 with regard to the $\mathrm{CN}(R, S)$ synthon created by Husson's group (Scheme 2), under the same experimental conditions. ${ }^{28}$ Following the literature data, ${ }^{29}$ we studied the methylation of 1 with iodomethane at $-78{ }^{\circ} \mathrm{C}$ in THF in presence of lithium diisopropylamine. First attempts were carried out on compound 1 without success. Therefore, it was decided to use the protected form, which was obtained by benzylation of the hydroxyl functions (Scheme 2 ). ${ }^{26}$

In contrast with the $\mathrm{CN}(R, S)$ synthon, the treatment of 2 with LDA in the presence of iodomethane gave two different products: the desired C5-alkylated compound $\mathbf{3}$ and an $\alpha$ cyanoenamine $\mathbf{4}$, in $10 \%$ and $30 \%$ yield, respectively. The major compound $\mathbf{4}$ was obtained through the elimination of the adjacent benzyloxy group. A careful analysis of 4 by ${ }^{1} \mathrm{H}$ - and ${ }^{13} \mathrm{C}$ NMR allowed the characterization of its structure. The typical NMR signals at $\delta=5.29 \mathrm{ppm}$ and at $\delta=112.5 \mathrm{ppm}$, observed in the ${ }^{1} \mathrm{H}$ and ${ }^{13} \mathrm{C}$ NMR spectra respectively, were attributed to the C6 position. 


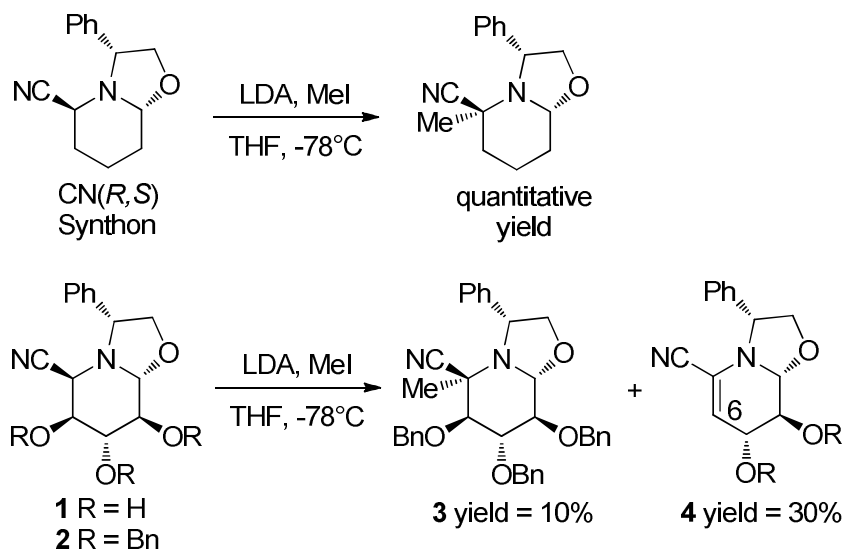

\section{Scheme 2}

As shown above, the $\beta$-elimination that took place was an obvious limitation for the access to the desired C-5-alkylated derivatives. This prompted us to change the protecting group for the $\beta$ hydroxyl function at the C-6 position to butane 2,3-bisacetal. This bisacetal was previously applied to various sugars ${ }^{30-32}$ or shikimic derivatives ${ }^{33-36}$ and in spite of the creation of two new stereogenic centers, the protection of vicinal diequatorial diols is described as being stereoselective. $^{10-37}$ Thus, the protection of hydroxyl functions of $\mathbf{1}$ with 2,2,3,3tetramethoxybutane, through stirring for a week in the presence of trimethylorthoformate and catalytic camphorsulfonic acid, has been performed. A 1:2 mixture (NMR determination) of the 6,7-butane-bisacetal 5 and its regioisomer $\mathbf{6}$ (Scheme 3) were obtained.

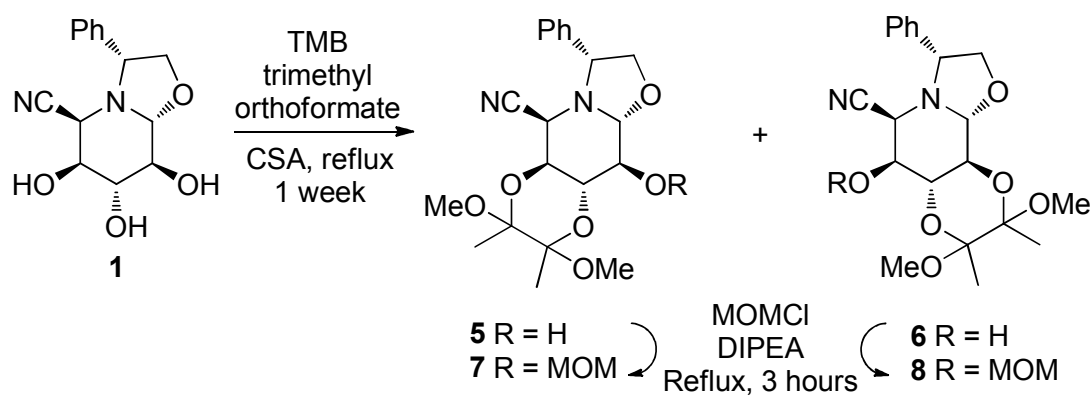

\section{Scheme 3}

Interestingly, an unexpected mixture of diastereoisomers on the butane 2,3-bisacetal protection for compounds $\mathbf{5}$ and $\mathbf{6}$, is formed. After crystallizing the diastereoisomers $\mathbf{6}$ in diethyl ether solution, this observation was confirmed by an X-Ray study. The stereochemistry of the protecting group is represented in Figure 2. 

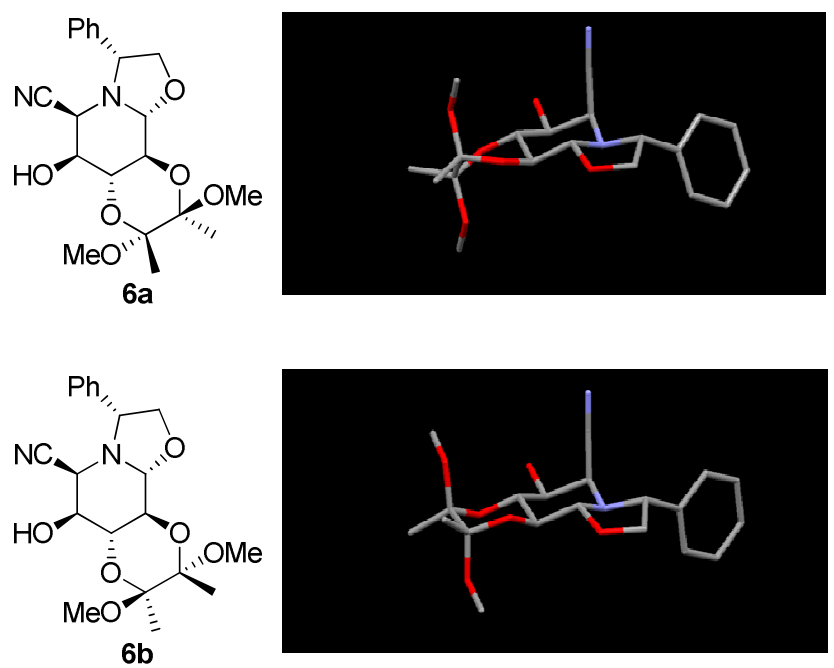

\section{Figure 2}

Structural features of interest of $\mathbf{6 a}$ and $\mathbf{6 b}$, which crystallizes in the non-centrosymmetric P21 2121 space group, are collected in Table 1.

Table 1. Lengths $(\AA)$, angles and torsion $\left(^{\circ}\right)$ of interest for complex $\mathbf{6 a}$ and $\mathbf{6 b}$

\begin{tabular}{lll}
\hline & $\mathbf{6 a}$ & $\mathbf{6 b}$ \\
\hline C7 O & $1.433(3)$ & $1.440(3)$. \\
C8 O & $1.437(4)$ & $1.441(4)$ \\
C2' O & $1.431(4)$ & $1.424(4)$ \\
C3' O & $1.436(4)$ & $1.448(4)$ \\
C2' C3' & $1.541(5)$ & $1.546(5)$ \\
& & \\
C7 O C2' & $115.7(3)$ & $111.9(2)$ \\
C7 C8 O & $110.9(2)$ & $107.8(2)$ \\
C3' O C8 & $115.3(2)$ & $113.1(3)$ \\
C2' C3' O & $111.1(3)$ & $111.1(3)$ \\
C3' C2' O & $110.7(2)$ & $110.5(3)$ \\
& & \\
C7 - O - C2' - C & $151.1(3)$ & $177.8(3)$ \\
C8 - O - C3' - C & $151.0(3)$ & $175.4(3)$ \\
C7 - O - C2' - O & $86.5(3)$ & $60.1(3)$ \\
C8 - O - C3' - O & $87.4(3)$ & $62.2(4)$ \\
\hline
\end{tabular}


The protecting group of $\mathbf{6 a}$ favors a twist boat conformation while a chair conformation is preferred for $\mathbf{6 b}$ (Figure 2). ${ }^{1} \mathrm{H}$ NMR studies indicated that after one week of reaction, compounds 6 were present in a mixture 80/20 with prevalence for the twist boat conformation of 6a. After two weeks of reaction, compounds $\mathbf{6}$ were still a mixture 80/20, but now with prevalence for the chair conformation of $\mathbf{6 b}$. This result suggests a kinetic and a thermodynamic form, with an equilibration to the more stable derivative under the acidic experimental conditions. To the best of our knowledge, no report exists for the characterization of the twist boat conformation with this stereochemistry, for the butane 2,3-bisacetal protection of vicinal diequatorial diols.

To pursue our work on the alkylation, the protection of compounds $\mathbf{5}$ and $\mathbf{6}$ on their free hydroxyl function was achieved by reacting with methoxymethyl chloride (MOMCl) in the presence of Hünig's base (Scheme 3). The structures of $\mathbf{7}$ and $\mathbf{8}$ were unambiguously deduced from their spectral data. Indeed, in the HMBC spectrum of compound 7 the cross peaks, observed between the triplet signal at $\delta=3.77$ of $\mathrm{H}-8$ proton and carbon signals at $\delta=91.7$ and $\delta$ = 95.9, were attributed to $\mathrm{C}-8 \mathrm{a}$ and MOM methylene $\mathrm{C}-1$ ' positions (Figure 3). Based on the HMBC correlation between H-6 and MOM methylene carbon (Figure 3), the structure of 8 was in full agreement with the proposed skeleton.

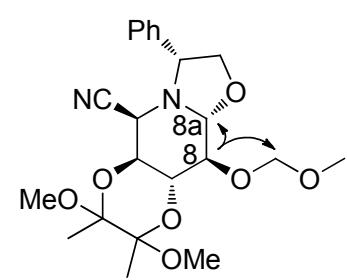

7

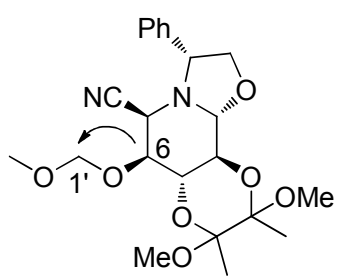

8

\section{Figure 3}

Initial attempts towards the stereoselective alkylation at the C-5 position of compounds 7 (mixture 50/50 of $\mathbf{7 a}$ and $\mathbf{7 b}$ confirmed by ${ }^{1} \mathrm{H}$ NMR), under classic conditions (LDA at - 78 ${ }^{\circ} \mathrm{C}$ ), were unsuccessful and only starting material was recovered. In contrast, when HMPA was added to the reaction and the temperature was gradually raised up to $-15^{\circ} \mathrm{C}$, lactams 9 were obtained in $60 \%$ yield as confirmed by NMR and MS spectroscopy $\left(\delta 162.7(\mathrm{CO}) ; \mathrm{MS}[\mathrm{M}+\mathrm{H}]^{+}\right.$ 424) and represented in Scheme 4. According to the literature, ${ }^{38}$ the lactam formation can be explained by in situ addition of oxygen from the solvent to an extremely oxygen sensitive anion. 


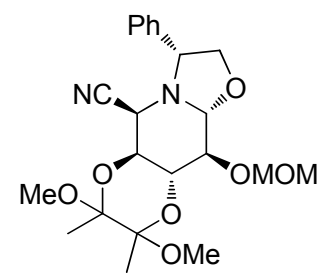

7

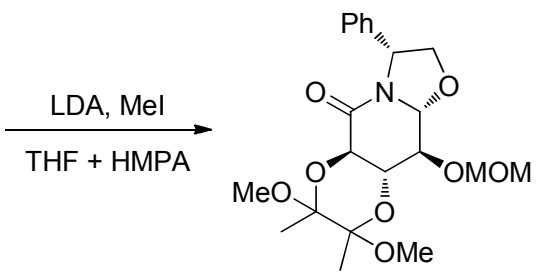

9 yield $=60 \%$

\section{Scheme 4}

This result showed that the anion formation occurred and that the 6,7-butane-bisacetal protection prevented the $\beta$-elimination. However, the alkylation reaction probably failed because of steric hindrance. In order to confirm this last hypothesis, we investigated the alkylation study on the less hindered 7,8-butane-bisacetals 8 (Scheme 5). Alkylation of 8 with iodomethane (LDA, THF, $-78^{\circ} \mathrm{C}$ ) led to the formation of the unexpected $\beta$-methylated- $\alpha$-cyanoenamines 10 (Scheme 5). These compounds were isolated in $77 \%$ yield after flash chromatography.

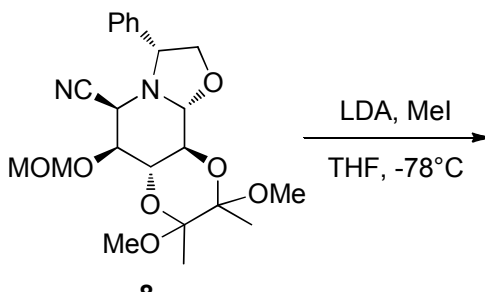

8

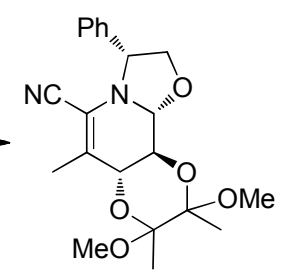

10 yield $=77 \%$

\section{Scheme 5}

These results suggested that the alkylation at $\alpha$-aminonitrile position was strongly dependant on the adjacent substituent. In the presence of a leaving group, the enamine formation is favored, whereas the presence of a sterically bulky group leads to the lactam derivative. Finally, we chose to use the non protected compounds $\mathbf{6}$ as a starting material, as seen in Scheme 6.

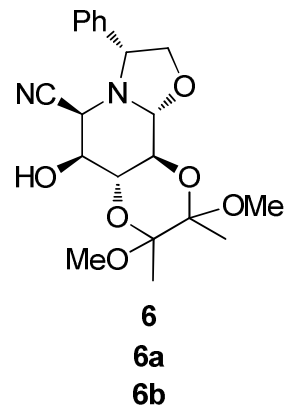

6b

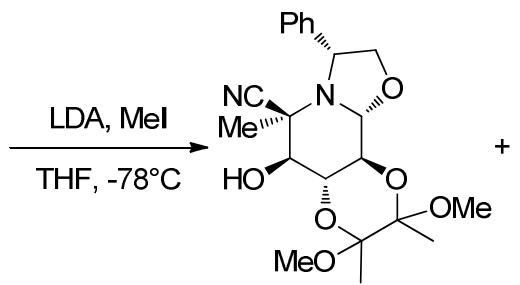

$11 \mathrm{a}$ yield $=50 \%$

$11 \mathrm{a}$ yield $=60 \%$

$11 \mathrm{~b}$ yield $=0 \%$

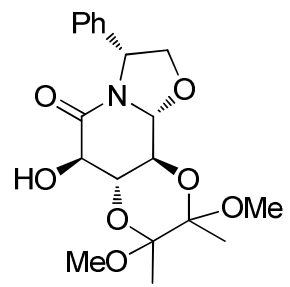

12 yield $=50 \%$

12a yield $=40 \%$

$12 b$ yield $=100 \%$

\section{Scheme 6}


Under similar conditions, compounds $\mathbf{6}$ are transformed into the corresponding alkylated compound 11a and the lactams 12 in equivalent yield, based on recovered starting material. Encouraged by this result, we decided to investigate the reactivity of the two diastereisomers separately. In this context, we first studied reactivity of compound $\mathbf{6 a}$. The alkylation reaction, following the same procedure, led to a mixture of 11a and 12a in $60 \%$ and $40 \%$ yield respectively. We then turned our attention to the second diasteroisomer $\mathbf{6 b}$. Reaction of $\mathbf{6 b}$ furnished the lactam $\mathbf{1 2 b}$ in quantitative yield. This result showed in our case the substratedependent reactivity concerning the butane 2,3-bisacetal protection group. The above observations led us to consider this method as a new and alternative access to alkylated or lactam compound, with increased yields.

\section{Conclusions}

In conclusion, we have demonstrated that compound $\mathbf{6 a}$ is a useful building block for alkylation reaction and that its diastereoisomer $\mathbf{6 b}$ allowed a selective access to the lactam scaffold. Considering the high potential of so-called "azasugars" for drug discovery, we want to extend this methodology to a new approach for the synthesis of indolizidine scaffold, through alkylation with suitable side chains and subsequent cyclization on the nitrogen after elimination of the chiral appendage. Efforts in this direction are currently being pursued in our laboratory.

\section{Experimental Section}

General. All reactions were carried out in dried glassware under an argon atmosphere. All solvents were purchased with an analytical grade from SDS. Acetone and chloroform were dried over molecular sieves. Reactions were monitored using thin-layer chromatography (TLC) carried out on $0.25 \mathrm{~mm}$ E. Merk silica gel plates (60F-254) using UV light as visualizing agent and sulfuric vanillin heated as developing agent. Flash chromatography was performed with silica gel CHROMATOGEL 60 (particle size 20-45 $\mu \mathrm{m}$ or 35-70 $\mu \mathrm{m}$ ) supplied by SDS. Yield refers to chromatography and spectroscopically pure compounds, unless otherwise noted. ${ }^{1} \mathrm{H}$ NMR spectra were recorded on a BRUCKER AC $300 \mathrm{MHz}$ spectrometer or a BRUCKER AVANCE $400 \mathrm{MHz}$ spectrometer. Chemical shifts are reported in ppm and coupling constants $(J)$ in $\mathrm{Hz}$. ${ }^{13} \mathrm{C}$ NMR spectra were recorded on a BRUCKER AC 300 spectrometer at $75 \mathrm{MHz}$. The assignments are based upon 1D and 2D spectra recorded using the following pulse sequences from the Bruker standard pulse program library: DEPT, COSY, HSQC, HMBC and NOESY. Mass spectra were measured with a ZQ 2000 Waters mass spectrometer (ESI). High resolution mass spectra were obtained on a Q-ToF1 ESI mass spectrometer (Waters). Infra-red spectra were recorded with a PerkinElmer spectrum 65 FT-IR spectrometer and wavelengths $(v)$ are given in

$\mathrm{cm}^{-1}$. Specific rotations were measured using a PerkinElmer model 341 polarimeter in a chloroform solution at $20{ }^{\circ} \mathrm{C}$. 
General procedure for the synthesis of 6,7,8-tribenzyloxy-5-methyl-3-phenylhexahydo-2Hoxazolo[3,2-a]pyridine-5-carbonitrile (3) and 7,8-bisbenzyloxy-3-phenyl-3,7,8,8atetrahydro-2H-oxazolo[3,2-a]pyridine-5-carbonitrile (4). To a solution of 2 (100 mg, 0.18 $\mathrm{mmol})$ in dry THF $(0.4 \mathrm{~mL})$ under argon $\left(-78{ }^{\circ} \mathrm{C}\right)$, was added dropwise a solution of LDA 1.6 $\mathrm{M}$ in dry THF $(0.32 \mathrm{~mL}, 0.72 \mathrm{mmol}, 4 \mathrm{eq})$. After one hour at $-78{ }^{\circ} \mathrm{C}$ iodomethane $(0.08 \mathrm{~mL}$, $1.52 \mathrm{mmol}, 8$ eq.) was added and the reaction stirred at this temperature for $3 \mathrm{~h}$. Then the temperature was raised up gradually to $0{ }^{\circ} \mathrm{C}$. The reaction was quenched with $\mathrm{NH}_{4} \mathrm{Cl}$ (sat) then extracted with ethyl acetate $(3 \times 40 \mathrm{~mL})$. The combined organic layers was dried over anhydrous $\mathrm{Na}_{2} \mathrm{SO}_{4}$, filtered and concentrated to dryness. After purification by chromatography on silica gel using as eluent a mixture of cyclohexane/ether $9 / 1,10 \mathrm{mg}$ of $3(10 \%)$ and $24 \mathrm{mg}$ of 4 (30\%) were afforded as yellow oils.

Data for 3. $R f: 0.41$ (cyclohexane/ether 8/2), IR $v \mathrm{~cm}^{-1}: 2229(\mathrm{CN}),{ }^{1} \mathrm{H}$ NMR (400 MHz, $\left.\mathrm{CDCl}_{3}\right): \delta 1.13\left(\mathrm{~s}, 3 \mathrm{H}, \mathrm{CH}_{3}\right), 3.35(\mathrm{~d}, 1 \mathrm{H}, J=9 \mathrm{~Hz}), 3.75(\mathrm{dd}, 1 \mathrm{H}, \mathrm{OH}, J=8 \mathrm{~Hz}, J=9 \mathrm{~Hz}), 3.86$ $(\mathrm{t}, 1 \mathrm{H}, J=9 \mathrm{~Hz}), 3.89(\mathrm{dd}, 1 \mathrm{H}, J=4.5 \mathrm{~Hz}, J=8.5 \mathrm{~Hz}), 4.10(\mathrm{dd}, 1 \mathrm{H}, J=4.5 \mathrm{~Hz}, J=8.5 \mathrm{~Hz})$, $4.37(\mathrm{t}, 1 \mathrm{H}, J=8.5 \mathrm{~Hz}), 4.40(\mathrm{~d}, 1 \mathrm{H}, J=8 \mathrm{~Hz}), 4.59(\mathrm{~d}, 1 \mathrm{H}, J=11.5 \mathrm{~Hz}), 4.78(\mathrm{~d}, 1 \mathrm{H}, J=11.1$ $\mathrm{Hz}), 4.80(\mathrm{~d}, 1 \mathrm{H}, J=11.5 \mathrm{~Hz}), 4.96(\mathrm{~d}, 1 \mathrm{H}, J=11.1 \mathrm{~Hz}), 4.99(\mathrm{~d}, 1 \mathrm{H}, J=11.5 \mathrm{~Hz}), 5.03(\mathrm{~d}, 1 \mathrm{H}$, $J=11.5 \mathrm{~Hz}), 7.15-7.50(\mathrm{~m}, 20 \mathrm{H}, \mathrm{H}-\mathrm{aro}) .{ }^{13} \mathrm{C} \mathrm{NMR}\left(75 \mathrm{MHz}, \mathrm{CDCl}_{3}\right): \delta 24.2\left(\mathrm{CH}_{3}\right), 59.9,61.3$, 74.0, 76.1, 76.3 (3 x CH $2-\mathrm{Ph}), 76.4,81.4,83.3,84.3,94.0,117.3(\mathrm{CN}), 126.9,127.5,127.8$, 128.0, 128.2, $\left.128.7\left(\mathrm{C}_{\mathrm{Ar}} \mathrm{H}\right), 137.5,138.1,138.5,143.5\left(\mathrm{C}_{\mathrm{Ar}}\right) . \mathrm{MS}\left(\mathrm{IC}, \mathrm{NH}_{3}\right): \mathrm{m} / z . \mathrm{M}+\mathrm{H}\right]^{+} 561$. HRMS (IC, $\mathrm{NH}_{3}$ ) $\mathrm{m} / z$ : calcd. for $\mathrm{C}_{36} \mathrm{H}_{36} \mathrm{~N}_{2} \mathrm{O}_{4} \mathrm{Na}[\mathrm{M}+\mathrm{Na}]^{+}$: 583.2573; found 583.2513.

Data for 4. $R f: 0.5$ (cyclohexane/ether 8/2), IR $v \mathrm{~cm}^{-1}: 2361(\mathrm{CN}),{ }^{1} \mathrm{H}$ NMR (400 $\mathrm{MHz}, \mathrm{CDCl}_{3}$ ): $\delta 3.84(\mathrm{dd}, 1 \mathrm{H}, J=7.5 \mathrm{~Hz}, J=8.5 \mathrm{~Hz}), 4.20(\mathrm{dd}, 1 \mathrm{H}, J=1.5 \mathrm{~Hz}, J=9 \mathrm{~Hz}), 4.35(\mathrm{dd}, 1 \mathrm{H}, J=6$ $\mathrm{Hz}, J=9 \mathrm{~Hz}), 4.38(\mathrm{ddd}, 1 \mathrm{H}, J=1 \mathrm{~Hz}, J=3 \mathrm{~Hz}, J=7.5 \mathrm{~Hz}), 4.6-4.7(\mathrm{~d}, 2 \mathrm{H}, J=11.5 \mathrm{~Hz}), 4.72$ $(\mathrm{dd}, 1 \mathrm{H}, J=1.5 \mathrm{~Hz}, J=6 \mathrm{~Hz}), 4.87(\mathrm{~d}, 1 \mathrm{H}, J=8.5 \mathrm{~Hz}), 4.9-5.1(\mathrm{~d}, 2 \mathrm{H}, J=11.5 \mathrm{~Hz}), 5.29(\mathrm{~d}, 1 \mathrm{H}$, $J=3 \mathrm{~Hz}$ ), 7.20-7.50 (m, $15 \mathrm{H}, \mathrm{H}$-aro). $\left.{ }^{13} \mathrm{C} \mathrm{NMR} \mathrm{(75} \mathrm{MHz,} \mathrm{CDCl}_{3}\right): \delta 60.9,72.3,74.1,74.9$ $\left(2 \mathrm{XCH}_{2} \mathrm{Ph}\right), 77.0,78.1,91.1,112.5,113.7(\mathrm{CN}), 127.3,127.8,128.1,128.3,128.5,128.6\left(\mathrm{C}_{\mathrm{Ar}} \mathrm{H}\right)$, 137.9, 138.2, $140.7\left(\mathrm{C}_{\mathrm{Ar}}\right)$. MS (IC, $\left.\mathrm{NH}_{3}\right): \mathrm{m} / z[\mathrm{M}+\mathrm{H}]^{+}$439. HRMS (IC, $\left.\mathrm{CH}_{4}\right) \mathrm{m} / z$ : calcd. for $\mathrm{C}_{28} \mathrm{H}_{26} \mathrm{~N}_{2} \mathrm{O}_{3}[\mathrm{M}+\mathrm{H}]^{+}$: 439.2022; found 439.1995.

General procedure for the synthesis of 5-hydroxy-7,8-dimethoxy-7,8-dimethyl-3-phenyloctahydro-1,6,9-trioxa-3a-aza-cyclopenta[a]naphthalene-4-carbonitrile (5)

and 9-hydroxy-6,7-dimethoxy-6,7-dimethyl-3-phenyl-octahydro-1,5,8-trioxa-3a-azacyclopenta $[\beta]$ naphthalene-4-carbonitrile (6). To a solution of trihydroxy derivative in dry $\mathrm{MeOH}(2 \mathrm{~mL})$, were added successively tetramethoxybutane (500 mg, $2.8 \mathrm{mmol}, 2.5$ eq.), trimethylorthoformate $(0.5 \mathrm{~mL})$, and camphorsulfonic acid $(0.1 \mathrm{mmol}, 0.1 \mathrm{eq}$.). The solution was refluxed for 1 week, then neutralized with $\mathrm{NaHCO}_{3}(220 \mathrm{mg})$. After extraction with $\mathrm{CH}_{2} \mathrm{Cl}_{2}(5 \mathrm{x}$ $30 \mathrm{~mL}$ ), the combined organic layers were washed with a saturated solution of $\mathrm{NaHCO}_{3}$, then dried over $\mathrm{Na}_{2} \mathrm{SO}_{4}$ and evaporated to dryness. After purification by flash chromatography on silica gel using as eluent a mixture of cyclohexane/ether 1/1, compound 5 was isolated as a colorless oil (65 mg, 15\%) and compound 6 as a white crystals (135 mg, 30\%). 
Data for 5. Mp: $264{ }^{\circ} \mathrm{C}, R_{f}: 0.35$ (cyclohexane/ether 1/1.5), IR $v \mathrm{~cm}^{-1}: 3456(\mathrm{OH}), 2229(\mathrm{CN})$, ${ }^{1} \mathrm{H}$ NMR (400 MHz, $\left.\mathrm{CDCl}_{3}\right): \delta 1.42,1.44\left(2 \mathrm{~s}, 6 \mathrm{H}, 2 \mathrm{CH}_{3}\right), 2.59(\mathrm{~d}, 1 \mathrm{H}, \mathrm{OH}, J=3 \mathrm{~Hz}), 3.37$, $3.41\left(2 \mathrm{~s}, 6 \mathrm{H}, 2 \mathrm{OCH}_{3}\right), 3.80-3.90(\mathrm{~m}, 1 \mathrm{H}), 3.88(\mathrm{t}, 1 \mathrm{H}, J=8 \mathrm{~Hz}), 3.98(\mathrm{~d}, 1 \mathrm{H}, J=5.5 \mathrm{~Hz}), 4.05$ $(\mathrm{t}, 1 \mathrm{H}, J=8 \mathrm{~Hz}), 4.17(\mathrm{dd}, 1 \mathrm{H}, J=8 \mathrm{~Hz}, J=10.5 \mathrm{~Hz}), 4.27(\mathrm{~d}, 1 \mathrm{H}, J=8 \mathrm{~Hz}), 4.28(\mathrm{~d}, 1 \mathrm{H}, J=$ $10.5 \mathrm{~Hz}), 4.39(\mathrm{t}, 1 \mathrm{H}, J=8 \mathrm{~Hz}), 7.30-7.40\left(\mathrm{~m}, 5 \mathrm{H}, \mathrm{C}_{6} \mathrm{H}_{5}\right) .{ }^{13} \mathrm{C} \mathrm{NMR}\left(75 \mathrm{MHz}, \mathrm{CDCl}_{3}\right): \delta 18.9$ $\left(2 \mathrm{CH}_{3}\right), 48.4\left(\mathrm{OCH}_{3}\right), 48.5\left(\mathrm{OCH}_{3}\right), 50.0,62.7,70.0,72.5,72.8,74.9,90.6,101.8,102.1\left(\mathrm{C}_{\mathrm{iv}}\right)$, $113.7(\mathrm{CN}), 127.8,129.1,129.2\left(\mathrm{C}_{\mathrm{Ar}} \mathrm{H}\right), 135.9\left(\mathrm{C}_{\mathrm{Ar}}\right) . \mathrm{MS}\left(\mathrm{IC}, \mathrm{NH}_{3}\right): \mathrm{m} / z .[\mathrm{M}+\mathrm{H}]^{+} 391 . \mathrm{HRMS}$ (IC, $\left.\mathrm{NH}_{3}\right) \mathrm{m} / z$ : calcd. for $\mathrm{C}_{20} \mathrm{H}_{26} \mathrm{~N}_{2} \mathrm{O}_{6} \mathrm{Na}[\mathrm{M}+\mathrm{Na}]^{+}$: 413.1689 ; found 413.1692

Data for 6. $R_{f}$ : 0.25 (cyclohexane/ether 1/1.5), IR $v \mathrm{~cm}^{-1}: 3450(\mathrm{OH}), 2229(\mathrm{CN}) .{ }^{1} \mathrm{H}$ NMR (400 $\left.\mathrm{MHz}_{\mathrm{CDCl}}\right): \delta 1.41,1.44\left(2 \mathrm{~s}, 6 \mathrm{H}, 2 \mathrm{CH}_{3}\right), 2.57(\mathrm{~d}, 1 \mathrm{H}, \mathrm{OH}, J=2.5 \mathrm{~Hz}), 3.41,3.45(2 \mathrm{~s}, 6 \mathrm{H}, 2$ $\left.\mathrm{OCH}_{3}\right), 3.69(\mathrm{t}, 1 \mathrm{H}, J=9 \mathrm{~Hz}), 3.84(\mathrm{dd}, 1 \mathrm{H}, J=9 \mathrm{~Hz}, J=5 \mathrm{~Hz}), 4.0-4.10(\mathrm{~m}, 1 \mathrm{H}), 4.27(\mathrm{~d}, 1 \mathrm{H}, J$ $=7.5 \mathrm{~Hz}), 4.33(\mathrm{dd}, 1 \mathrm{H}, J=11 \mathrm{~Hz}, J=5 \mathrm{~Hz}), 4.30-4.40(\mathrm{~m}, 2 \mathrm{H}), 7.30-7.40\left(\mathrm{~m}, 5 \mathrm{H}, \mathrm{C}_{6} \mathrm{H}_{5}\right) .{ }^{13} \mathrm{C}$ NMR (75 MHz, $\left.\mathrm{CDCl}_{3}\right): \delta$ 18.7, $18.8\left(2 \mathrm{CH}_{3}\right), 47.8\left(\mathrm{OCH}_{3}\right), 48.3\left(\mathrm{OCH}_{3}\right), 48.9,62.7,69.8$, 72.9, 73.5, 74.3, 91.6, 101.7, $102.0\left(\mathrm{C}_{\mathrm{iv}}\right), 113.9(\mathrm{CN}), 127.8,129.0,129.1\left(\mathrm{C}_{\mathrm{Ar}} \mathrm{H}\right), 135.7\left(\mathrm{C}_{\mathrm{Ar}}\right)$. MS (IC, $\left.\mathrm{NH}_{3}\right): m / z[\mathrm{M}+\mathrm{H}]^{+} 391$.

Crystallographic data for 6a. C20 H26 N2 O6, orthorhombic, P21 2121 , colorless, a = 6.9853(5) $\AA, b=10.5499(6) \AA, c=26.538(2) \AA, V=1955.7(2) \AA^{3}, T=293(2) K, Z=4$, Final $R$ $(\mathrm{I}>2 \sigma(\mathrm{I})): \mathrm{R}_{1}=0.0463, \mathrm{wR}_{2}=0.0986, \mathrm{GOF}=1.070$. Structural information for $\mathbf{6 a}$ has been deposited with CCDC as 845878 .

Crystallographic data for 6b. C20 H26 N2 O6, orthorhombic, P21 2121 , colorless, a = 6.6354(1) $\AA, b=12.4162(2)(6) \AA, c=24.790(2) \AA, V=2042.4(2) \AA^{3}, T=293(2) \mathrm{K}, \mathrm{Z}=4$, Final $\mathrm{R}(\mathrm{I}>2 \sigma(\mathrm{I})): \mathrm{R}_{1}=0.0441, \mathrm{wR}_{2}=0.0834, \mathrm{GOF}=1.154$. Structural information for $\mathbf{6 b}$ has been deposited with CCDC as 845879 .

General procedure for the synthesis of 6,7-dimethoxy-9-methoxymethoxy-6,7-dimethyl-3phenyl-octahydro-1,5,8-trioxa-3a-aza-cyclopenta[a]naphthalene-4-carbonitrile (7) and 7,8-dimethoxy-5-methoxymethoxy-7,8-dimethyl-3-phenyl-octahydro-1,6,9-trioxa-3a-azacyclopenta[a]naphthalene-4-carbonitrile (8). To a solution of 5 (50 mg, $0.13 \mathrm{mmol})$ or of 6 $(65 \mathrm{mg}, 0.15 \mathrm{mmol})$ in dry $\mathrm{CHCl}_{3}(1 \mathrm{~mL})$, was added at $0{ }^{\circ} \mathrm{C}$ methoxymethyl chloride $(26 \mu \mathrm{L}$, 2.5 eq., $0.32 \mathrm{mmol})$ and $N$ - $N$-diisopropylamine $(68 \mu \mathrm{L}, 3$ eq., $0.4 \mathrm{mmol})$. The resulting mixture was refluxed for $3 \mathrm{~h}$, then let to cool down to $\mathrm{rt}$. The solution was quenched with water and extracted with dichloromethane $(5 \times 30 \mathrm{~mL})$. The combined organic layers were dried over anhydrous $\mathrm{Na}_{2} \mathrm{SO}_{4}$, filtered and evaporated to dryness. After purification by chromatography on silica gel using as eluent a mixture of cyclohexane/ether 1/0.5) the compound 7 was recovered as a colorless oil $(7.5 \mathrm{mg}, 77 \%)$.

Data for 7. $R_{f}: 0.60$ (cyclohexane/ether 1/1.5), ${ }^{1} \mathrm{H}$ NMR $\left(400 \mathrm{MHz}, \mathrm{CDCl}_{3}\right): \delta 1.34,1.40(2 \mathrm{~s}$, $\left.6 \mathrm{H}, 2 \mathrm{CH}_{3}\right), 3.40,3.42,3.52\left(3 \mathrm{~s}, 9 \mathrm{H}, 3 \mathrm{OCH}_{3}\right), 3.77(\mathrm{t}, 1 \mathrm{H}, J=8 \mathrm{~Hz}), 3.83(\mathrm{t}, 1 \mathrm{H}, J=8 \mathrm{~Hz}), 3.96$ $(\mathrm{d}, 1 \mathrm{H}, J=5.5 \mathrm{~Hz}), 3.99$ (t, $1 \mathrm{H}, J=8 \mathrm{~Hz}), 4.28(\mathrm{~d}, 1 \mathrm{H}, J=8 \mathrm{~Hz}), 4.30-4.40(\mathrm{~m}, 2 \mathrm{H}, \mathrm{H}-8), 4.46$ $(\mathrm{t}, 1 \mathrm{H}, J=10 \mathrm{~Hz}), 4.85-5.00\left(2 \mathrm{~d}, 2 \mathrm{H}, \mathrm{OCH}_{2} \mathrm{O}, J=6.5 \mathrm{~Hz}\right), 7.20-7.50\left(\mathrm{~m}, 5 \mathrm{H}, \mathrm{C}_{6} \mathrm{H}_{5}\right) .{ }^{13} \mathrm{C} \mathrm{NMR}$ $\left(75 \mathrm{MHz}, \mathrm{CDCl}_{3}\right): \delta$ 18.7, $19.0\left(2 \mathrm{CH}_{3}\right), 48.2,48.8,55.4\left(3 \mathrm{XOCH}_{3}\right), 62.5,70.1,72.3,74.3,75.4$, 
91.7, $95.9\left(\mathrm{OCH}_{2} \mathrm{O}\right), 101.5,101.8\left(\mathrm{C}_{\mathrm{iv}}\right), 113.9(\mathrm{CN}), 127.7$ 129.0, $129.2\left(\mathrm{C}_{\mathrm{Ar}} \mathrm{H}\right), 135.9\left(\mathrm{C}_{\mathrm{Ar}}\right) . \mathrm{MS}$ $\left(\mathrm{IC}, \mathrm{NH}_{3}\right): m / z[\mathrm{M}+\mathrm{H}]^{+}$435. HRMS $\left(\mathrm{IC}, \mathrm{NH}_{3}\right) \mathrm{m} / z$ : calcd. for $\mathrm{C}_{22} \mathrm{H}_{30} \mathrm{~N}_{2} \mathrm{O}_{7} \mathrm{Na}[\mathrm{M}+\mathrm{Na}]^{+}$: 457.1951; found 457.1903.

Data for 8. $R_{f}$ : 0.40 (cyclohexane/ether 1/1), IR $v \mathrm{~cm}^{-1}: 2229(\mathrm{CN}) .{ }^{1} \mathrm{H}$ NMR (400 MHz, $\left.\mathrm{CDCl}_{3}\right)$ : $\delta 1.42,1.43\left(2 \mathrm{~s}, 6 \mathrm{H}, 2 \mathrm{CH}_{3}\right), 3.37,3.40,3.41\left(3 \mathrm{~s}, 9 \mathrm{H}, 3 \mathrm{OCH}_{3}\right), 3.81(\mathrm{dd}, 1 \mathrm{H}, J=5.5 \mathrm{~Hz}, J=10$ $\mathrm{Hz}), 3.86(\mathrm{t}, 1 \mathrm{H}, J=8 \mathrm{~Hz}), 4.00(\mathrm{~d}, 1 \mathrm{H}, J=5.5 \mathrm{~Hz}), 4.05(\mathrm{t}, 1 \mathrm{H}, J=8 \mathrm{~Hz}), 4.19(\mathrm{dd}, 1 \mathrm{H}, J=8$ $\mathrm{Hz}, J=10 \mathrm{~Hz}), 4.24(\mathrm{~d}, 1 \mathrm{H}, J=8 \mathrm{~Hz}), 4.37(\mathrm{t}, 1 \mathrm{H}, J=10 \mathrm{~Hz}), 4.38(\mathrm{t}, 1 \mathrm{H}, J=8 \mathrm{~Hz}), 4.70,4.79$ $\left(2 \mathrm{~d}, 2 \mathrm{H}, \mathrm{OCH}_{2} \mathrm{O}, J=7 \mathrm{~Hz}\right), 7.20-7.50\left(\mathrm{~m}, 5 \mathrm{H}, \mathrm{C}_{6} \mathrm{H}_{5}\right) .{ }^{13} \mathrm{C} \mathrm{NMR}\left(75 \mathrm{MHz}, \mathrm{CDCl}_{3}\right): \delta 18.8,19.0$ $\left(2 \mathrm{CH}_{3}\right), 48.1\left(\mathrm{OCH}_{3}\right), 48.3\left(\mathrm{OCH}_{3}\right), 49.6,56.2,62.8,71.8,72.6,74.6,74.9,90.2,96.8\left(\mathrm{OCH}_{2} \mathrm{O}\right)$, 101.4, 101.7 (C $\left.\mathrm{C}_{\mathrm{iv}}\right), 113.6(\mathrm{CN}), 127.8,129.1,129.2\left(\mathrm{C}_{\mathrm{Ar}} \mathrm{H}\right), 136.0\left(\mathrm{C}_{\mathrm{Ar}}\right) . \mathrm{MS}\left(\mathrm{IC}, \mathrm{NH}_{3}\right): \mathrm{m} / z[\mathrm{M}+$ $\mathrm{Na}]^{+}$457. HRMS (IC, $\mathrm{NH}_{3}$ ) $m / z$ : calcd. for $\mathrm{C}_{22} \mathrm{H}_{30} \mathrm{~N}_{2} \mathrm{O}_{7} \mathrm{Na}[\mathrm{M}+\mathrm{Na}]^{+}$: 457.1951; found 457.1929.

Procedure for the synthesis of 6,7-dimethoxy-9-methoxymethoxy-6,7-dimethyl-3-phenyloctahydro-1,5,8-trioxa-3a-aza-cyclopenta[a]naphthalene-4-one (9). To a solution of 7 (13 $\mathrm{mg}, 0.03 \mathrm{mmol})$ in dry THF $(100 \mu \mathrm{L})$ under argon and at $-78^{\circ} \mathrm{C}$, was added dropwise a solution of LDA $2.5 \mathrm{M}$ in dry THF $(110 \mu \mathrm{L}, 0.12 \mathrm{mmol}, 4 \mathrm{eq})$ and HMPA (26 $\mu \mathrm{L}, 0.15 \mathrm{mmol}, 5 \mathrm{eq})$. After $20 \mathrm{~min}$ at $-78^{\circ} \mathrm{C}$ iodomethane $(15 \mu \mathrm{L}, 0.24 \mathrm{mmol}, 8$ eq. $)$ was added and the reaction was stirred at this temperature for $3 \mathrm{~h} 30$. Then the temperature was raised up gradually to $-15^{\circ} \mathrm{C}$ for $2 \mathrm{~h}$. The reaction was quenched with $\mathrm{NH}_{4} \mathrm{Cl}$ (sat) then extracted with $\mathrm{CH}_{2} \mathrm{Cl}_{2}$. The organic layer was dried over anhydrous $\mathrm{Na}_{2} \mathrm{SO}_{4}$, filtered and concentrated to dryness. After purification by chromatography on silica gel using as eluent a mixture of cyclohexane/ether 1/0.3, compound 9 was afforded as a yellow oil ( $8 \mathrm{mg}, 60 \%)$.

Data for 9. $R_{f}: 0.5\left(\mathrm{CH}_{2} \mathrm{Cl}_{2} / \mathrm{MeOH} 9 / 1\right) .{ }^{1} \mathrm{H} \mathrm{NMR}\left(400 \mathrm{MHz}, \mathrm{CDCl}_{3}\right): \delta 1.31,1.49(2 \mathrm{~s}, 6 \mathrm{H}, 2$ $\left.\mathrm{CH}_{3}\right)$, 3.20-3.60 (m, 9H, $\left.3 \mathrm{OCH}_{3}\right), 4.05(\mathrm{t}, 1 \mathrm{H}, J=8.5 \mathrm{~Hz}), 4.10-4.20(\mathrm{~m}, 3 \mathrm{H}), 4.40-4.50(\mathrm{~m}$, $1 \mathrm{H}), 4.77(\mathrm{~d}, 1 \mathrm{H}, J=7.5 \mathrm{~Hz}), 4.86(\mathrm{t}, 1 \mathrm{H}, J=8 \mathrm{~Hz}), 4.94,5.01\left(2 \mathrm{~d}, 2 \mathrm{H}, \mathrm{OCH}_{2} \mathrm{O}, J=7 \mathrm{~Hz}\right), 7.20-$ $7.60\left(\mathrm{~m}, 5 \mathrm{H}, \mathrm{C}_{6} \mathrm{H}_{5}\right) .{ }^{13} \mathrm{C} \mathrm{NMR}\left(75 \mathrm{MHz} \mathrm{CDCl}_{3}\right): \delta$ 17.5, $18.6\left(2 \mathrm{CH}_{3}\right), 47.8,48.2$, 55.6 $\left(3 \mathrm{XOCH}_{3}\right), 58.1,70.6,72.7,74.7,74.8,89.8,96.1\left(\mathrm{O}_{-} \mathrm{CH}_{2}-\mathrm{O}\right), 99.6,101.8\left(\mathrm{C}_{\mathrm{iv}}\right), 126.8128 .0$, $128.9\left(\mathrm{C}_{\mathrm{Ar}} \mathrm{H}\right), 139.9\left(\mathrm{C}_{\mathrm{Ar}}\right), 162.7(\mathrm{CO}) . \mathrm{MS}\left(\mathrm{IC}, \mathrm{NH}_{3}\right): m / z[\mathrm{M}+\mathrm{H}]^{+} 424$.

General procedure for the alkylation reaction of 7,8-dimethoxy-5,7,8-trimethyl-1,3-phenyl2,3,7,8,9a,9b-hexahydro-5aH-1,6,9-trioxa-3a-aza- cyclopenta[a]naphthalene-4-carbonitrile (10), of $(2 R, 3 R, 4 \mathrm{a} R, 5 S, 6 R, 8 R, 10 \mathrm{a} R, 10 \mathrm{~b} S)$-5-hydroxy-2,3-dimethoxy-2,3,6-trimethyl-8phenyloctahydro-2H-[1,4]dioxino[2,3-c] oxazolo[3,2-a]pyridine-6-carbonitrile (11a), of $(2 R, \quad 3 R, \quad 4 \mathrm{a} R, \quad 5 S, \quad 8 R, \quad 10 \mathrm{a} R, \quad 10 \mathrm{~b} S)$-5-hydroxy-2,3-dimethoxy-2,3-dimethyl-8phenylhexahydro-2H-[1,4]dioxino[2,3-c] oxazolo[3,2-a]pyridin-6(3H)-one (12a) and of $(2 R, 3 R, 4 \mathrm{a} R, 5 S, 8 R, 10 \mathrm{a} R, 10 \mathrm{~b} S)$-5-hydroxy-2,3-dimethoxy-2,3-dimethyl-8phenylhexahydro- $2 H$-[1,4]dioxino[2,3-c]oxazolo[3,2-a]pyridin-6(3H)-one $\quad(12 b)$. To a solution of 8,6 , $6 \mathbf{a}$ or $\mathbf{6 b}(0.025 \mathrm{mmol})$ in dry THF $(40 \mu \mathrm{L})$ under argon $\left(-78{ }^{\circ} \mathrm{C}\right)$, was added dropwise a solution of LDA $2.5 \mathrm{M}$ in dry THF $(0.1 \mathrm{mmol}, 4 \mathrm{eq})$. After $15 \mathrm{~min}$. at $-78{ }^{\circ} \mathrm{C}$, iodomethane $(0.35 \mathrm{mmol}, 14$ eq.) was added and the reaction was stirred at this temperature for 3 h. The reaction was quenched with $\mathrm{NH}_{4} \mathrm{Cl}$ (sat) then extracted with $\mathrm{CH}_{2} \mathrm{Cl}_{2}(3 \times 30 \mathrm{~mL})$. The 
combined organic layers were dried over anhydrous $\mathrm{Na}_{2} \mathrm{SO}_{4}$, filtered and concentrated to dryness. Purification by chromatography on silica gel was then performed using as eluent a mixture of cyclohexane/ether, 9/1.

Data for 10. $R_{f}: 0.5$ (cyclohexane/ether 1/1), IR $v \mathrm{~cm}^{-1}: 2223(\mathrm{CN}) .{ }^{1} \mathrm{H}$ NMR $(400 \mathrm{MHz}$, $\left.\mathrm{CDCl}_{3}\right): \delta 1.11-1.30\left(3 \mathrm{~s}, 6 \mathrm{H}, 2 \mathrm{CH}_{3}\right), 1.62\left(\mathrm{~s}, 3 \mathrm{H}, \mathrm{CH}_{3}\right), 2.90-3.20\left(3 \mathrm{~s}, 6 \mathrm{H}, 2 \mathrm{OCH}_{3}\right), 3.90-4.00$ $(\mathrm{m}, 1 \mathrm{H}), 4.10(\mathrm{dd}, 1 \mathrm{H}, J=6 \mathrm{~Hz}, J=8 \mathrm{~Hz}), 4.17(\mathrm{t}, 1 \mathrm{H}, J=9 \mathrm{~Hz}), 4.49(\mathrm{t}, 1 \mathrm{H}, J=6 \mathrm{~Hz}, J=6.5$ $\mathrm{Hz}), 4.56(\mathrm{~d}, 1 \mathrm{H}, J=10 \mathrm{~Hz}), 4.69(\mathrm{~d}, 1 \mathrm{H}, J=9 \mathrm{~Hz}), 7.10-7.30\left(\mathrm{~m}, 5 \mathrm{H}, \mathrm{C}_{6} \mathrm{H}_{5}\right) \cdot{ }^{13} \mathrm{C}$ NMR $(75$ $\left.\mathrm{MHz}, \mathrm{CDCl}_{3}\right): \delta 14.1\left(\mathrm{CH}_{3}\right), 19.0\left(2 \mathrm{CH}_{3}\right), 47.8\left(2 \mathrm{OCH}_{3}\right), 62.3,69.6,70.5,74.6,89.0,102.1$ $\left(\mathrm{C}_{\mathrm{iv}}\right), 111.0,112.9,114.1(\mathrm{CN}), 128-129\left(\mathrm{C}_{\mathrm{Ar}} \mathrm{H}\right), 140.4\left(\mathrm{C}_{\mathrm{Ar}}\right) . \mathrm{MS}\left(\mathrm{IC}, \mathrm{NH}_{3}\right): m / z[\mathrm{M}+\mathrm{Na}]^{+} 409$. HRMS (IC, $\mathrm{NH}_{3}$ ) $\mathrm{m} / z$ : calcd. for $\mathrm{C}_{21} \mathrm{H}_{26} \mathrm{~N}_{2} \mathrm{O}_{5} \mathrm{Na}[\mathrm{M}+\mathrm{Na}]^{+}$: 409.1739; found 409.1745.

Data for 11a. $R_{f} 0.4$ (cyclohexane/ether, $\left.1: 1\right),[\alpha]_{\mathrm{D}}=-109(c 0.58, \mathrm{MeOH})$; IR (film) $\mathrm{cm}^{-1}: 3484$ $(\mathrm{OH}) .{ }^{1} \mathrm{H}$ NMR $\left(\mathrm{CDCl}_{3}\right) \delta 1.20\left(\mathrm{~s}, 3 \mathrm{H}, \mathrm{CH}_{3}\right), 1.42\left(\mathrm{~s}, 3 \mathrm{H}, \mathrm{CH}_{3}\right), 1.44\left(\mathrm{~s}, 3 \mathrm{H}, \mathrm{CH}_{3}\right), 3.38(\mathrm{~s}, 3 \mathrm{H}$, $\left.\mathrm{OCH}_{3}\right), 3.41\left(\mathrm{~s}, 3 \mathrm{H}, \mathrm{OCH}_{3}\right), 3.88(\mathrm{dd}, 1 \mathrm{H}, J=4.7 ; 8.6 \mathrm{~Hz}), 4.13(\mathrm{dd}, J=4.7 ; 8.6 \mathrm{~Hz}, 1 \mathrm{H}), 4.21-$ $4.30(\mathrm{~m}, 3 \mathrm{H}), 4.36(\mathrm{t}, J=8.6 \mathrm{~Hz}, 1 \mathrm{H}), 7.22-7.38(\mathrm{~m}, 5 \mathrm{H}$ arom $) .{ }^{13} \mathrm{C}$ NMR $\left(\mathrm{CDCl}_{3}\right): \delta 19.1$ $\left(2 \mathrm{XCH}_{3}\right), 23.6\left(\mathrm{CH}_{3}\right), 48.4\left(\mathrm{OCH}_{3}\right), 48.5\left(\mathrm{OCH}_{3}\right), 60.1,61.5,71.9,73.0,76.1,76.6,92.0,101.8$, 102.2, $117.3(\mathrm{CN}), 127.1,128.0,129.0\left(\mathrm{CH}\right.$ arom), 143.2 (C $\mathrm{C}_{\mathrm{IV}}$ arom). HRMS (ESI): calcd for $\mathrm{C}_{21} \mathrm{H}_{29} \mathrm{~N}_{2} \mathrm{O}_{6}[\mathrm{M}+\mathrm{H}]^{+}:$405.2026; found 405.2026. calcd. for $\mathrm{C}_{20} \mathrm{H}_{27} \mathrm{NO}_{6} \mathrm{Na}[\mathrm{M}-\mathrm{HCN}+\mathrm{Na}]^{+}$: 400.1736; found 401.1737 .

Data for 12a. $R_{f} 0.6$ (dichloromethane/methanol, 9:1), $[\alpha]_{\mathrm{D}}=-26(c 1.01, \mathrm{MeOH}) ; \mathrm{IR}(\mathrm{film}) \mathrm{cm}$ ${ }^{1}: 3390(\mathrm{OH}), 1668(\mathrm{CO}) ;{ }^{1} \mathrm{H} \mathrm{NMR}\left(\mathrm{CDCl}_{3}\right) \delta 1.45\left(\mathrm{~s}, 3 \mathrm{H}, \mathrm{CH}_{3}\right), 1.47\left(\mathrm{~s}, 3 \mathrm{H}, \mathrm{CH}_{3}\right), 3.40(\mathrm{~s}, 6 \mathrm{H}$, $\left.\mathrm{OCH}_{3}\right), 3.91(\mathrm{~d}, J=8.2 \mathrm{~Hz}, 1 \mathrm{H}), 4.20(\mathrm{~d}, J=9.7 \mathrm{~Hz}, 1 \mathrm{H}), 4.27-4.35(\mathrm{~m}, 1 \mathrm{H}), 4.36-4.69(\mathrm{~m}, 2 \mathrm{H})$, $4.79(\mathrm{~d}, J=6.7 \mathrm{~Hz}, 1 \mathrm{H}), 4.88(\mathrm{~d}, J=6.5 \mathrm{~Hz}, 1 \mathrm{H}), 7.20-7.50(\mathrm{~m}, 5 \mathrm{H}$ arom $) .{ }^{13} \mathrm{C} \mathrm{NMR}\left(\mathrm{CDCl}_{3}\right)$ : $\delta 18.8\left(\mathrm{CH}_{3}\right), 19.0\left(\mathrm{CH}_{3}\right), 48.7\left(\mathrm{OCH}_{3}\right), 48.8\left(\mathrm{OCH}_{3}\right), 58.7,70.8,71.0,72.7,75.2,89.3,101.6$,

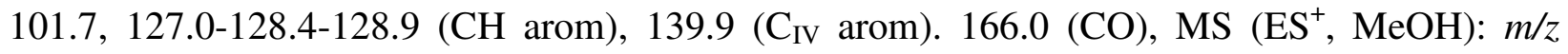
$[\mathrm{M}+\mathrm{Na}]^{+} 402 ;[2 \mathrm{M}+\mathrm{Na}]^{+}$780. HRMS (ES) : calcd. for $\mathrm{C}_{19} \mathrm{H}_{25} \mathrm{NO}_{7} \mathrm{Na}[\mathrm{M}+\mathrm{Na}]^{+}: 402.1529$; found 402.1526 .

Data for 12b. $R_{f} 0.62$ (dichloromethane/methanol, 9:1); IR (film) $\mathrm{cm}^{-1}: 3390(\mathrm{OH}), 1670(\mathrm{CO})$; ${ }^{1} \mathrm{H} \mathrm{NMR}\left(\mathrm{CDCl}_{3}\right) \delta 1.38\left(\mathrm{~s}, 3 \mathrm{H}, \mathrm{CH}_{3}\right), 1.40\left(\mathrm{~s}, 3 \mathrm{H}, \mathrm{CH}_{3}\right), 3.41\left(\mathrm{~s}, 6 \mathrm{H}, \mathrm{OCH}_{3}\right), 4.05(\mathrm{~m}, 2 \mathrm{H}), 4.18$ $(\mathrm{t}, J=8.1 \mathrm{~Hz}, 1 \mathrm{H}), 4.27-4.35(\mathrm{~m}, 1 \mathrm{H}), 4.39-4.49(\mathrm{~m}, 2 \mathrm{H}), 4.95-4.97(\mathrm{~m}, 2 \mathrm{H}), 7.20-7.50(\mathrm{~m}, 5 \mathrm{H}$ arom). ${ }^{13} \mathrm{C} \mathrm{NMR}\left(\mathrm{CDCl}_{3}\right): \delta 17.6\left(\mathrm{CH}_{3}\right), 17.8 .0\left(\mathrm{CH}_{3}\right), 48.4\left(\mathrm{OCH}_{3}\right), 48.6\left(\mathrm{OCH}_{3}\right), 58.7,68.5$, 68.9, 70.5, 75.1, 87.7, 99.4, 99.06, 126.7-128.3-131.0 (CH arom), $139.9\left(\mathrm{C}_{\mathrm{IV}}\right.$ arom). $166.2(\mathrm{CO})$. MS $\left(\mathrm{ES}^{+}, \mathrm{MeOH}\right): \mathrm{m} / z,[\mathrm{M}+\mathrm{Na}]^{+}$402; $[2 \mathrm{M}+\mathrm{Na}]^{+}$780. HRMS HRMS (ES): calcd. for $\mathrm{C}_{19} \mathrm{H}_{25} \mathrm{NO}_{7} \mathrm{Na}[\mathrm{M}+\mathrm{Na}]^{+}:$402.1529; found 402.1526 .

\section{Acknowledgements}

Financial supports of this work by Université Paris Descartes, Sorbonne Paris Cité (grant to Alicia M. Dilmaç) is gratefully acknowledged. We thank Drs. Xavier Cachet, Andy Gum and François-Hugues Porée for proofreading of our manuscript. 


\section{References}

1. Legler G. Iminosugars as Glycosidase inhibitors-Nojirimycin and Beyond; Stüt, A. E. Ed.; Wiley-VCH: Weinheim, 1999; pp 31-66.

2. Lillelund, V. H.; Jensen, H. H.; Liang, X.; Bols, M. Chem. Rev. 2002, 102, 515. http://dx.doi.org/10.1021/cr000433k

3. Martin, O. R.; Compain. P. Curr. Top. Med. Chem. 2003, 3, 541.

4. Borges de Melo E.; da Silveira Gomes A.; Carvalho I. Tetrahedron 2006, 62, 10277. http://dx.doi.org/10.1016/j.tet.2006.08.055

5. Compain, P.; Martin, O. R. Iminosugars: From Synthesis to Therapeutic Applications; Wiley-VCH: Weiheim, 2007.

6. Wrodnigg, T. M., Steiner, A. J.; Ueberbacher, B. J. Anti-Cancer Agents Med. Chem. 2008, 8, 77. http://dx.doi.org/10.2174/187152008783330851

7. Oulaïdi, F.; Front-Deschamps, S.; Galienne, E.; Leselier, E.; Ikeda, K.; Asano, N.; Compain, P.; Martin, O. R. Chem. Med. Chem. 2011, 6, 353.

http://dx.doi.org/10.1002/cmdc.201000469

8. Home, G.; Wilson, F. X.; Tinsley, J.; Williams, D. H.; Storer, R. Drug Discov. Today 2011, $16,107$. http://dx.doi.org/10.1016/i.drudis.2010.08.017

9. Home, G.; Wilson, F. X. Prog. Med. Chem. 2011, 50, 135. http://dx.doi.org/10.1016/B978-0-12-381290-2.00004-5 PMid:21315930

10. Zingfei, H.; Xinshan, Y.; Zhongguo K. Kexue : Huaxue 2012, 42, 1732.

11. Simmonds, M. S. J.; Kite, G. C.; Porter, E. A. Iminosugars as Glycosidase inhibitorsNojirimycin and Beyond; Stüt, A. E. Ed.; Wiley-VCH:Weinheim, 1999, pp 8-30.

12. Watson, A. A.; Fleet, G. W. J.; Asano, N.; Molyneux, R. J.; Nash, R. J. Phytochem. 2001, 56, 265.

http://dx.doi.org/10.1016/S0031-9422(00)00451-9

13. Asano, N. Curr. Top. Med. Chem. 2003, 3, 471. http://dx.doi.org/10.2174/1568026033452438 PMid:12570862

14. Kato, A.; Kato, N.; Miyauchi, S.; Minoshima, Y.; Adachi, I.; Ikeda, K.; Asano, N.; Watson, A. A.; Nash, R. J. Phytochem. 2008, 69, 1261. http://dx.doi.org/10.1016/j.phytochem.2007.11.018

15. Winchester, B. G. Tetrahedron : Asymmetry 2009, 20, 645. http://dx.doi.org/10.1016/j.tetasy.2009.02.048

16. Rodriguez-Sanchez, S.; Ruiz-Aceituno, L.; Sanz, M. L.; Soria, A. C. J. Agric. Food Chem. 2013, 61, 4539 . http://dx.doi.org/10.1021/jf305049k

17. Pearson, M. S. M.; Mathé-Allainmat, M.; Frageas, V.; Lebreton, J. Eur. J. Org. Chem. 2005 , 2159. http://dx.doi.org/10.1002/ejoc.200400823 
18. Kadouri-Puchot, C.; Comesse, S. Amino Acids 2005, 29, 101. http://dx.doi.org/10.1007/s00726-005-0193-X

19. Compain, P.; Chagnault, V.; Martin, O. R. Tetrahedron : Asymmetry 2009, 20, 672. http://dx.doi.org/10.1016/j.tetasy.2009.03.031

20. Davis, B. G. Tetrahedron : Asymmetry 2009, 20, 652. http://dx.doi.org/10.1016/j.tetasy.2009.03.013

21. Zhang, G. L.; Zheng, X. J.; Zhang, L. H.; Ye, X. S. Med. Chem. Comm. 2011, 2, 909. http://dx.doi.org/10.1039/c1md00098e

22. Wang, G. N.; Xiong, Y. L.; Ye, J.; Zang, G. L.; Ye, X. S., Med. Chem. Lett. 2011, 2, 682. http://dx.doi.org/10.1021/ml2000998

23. Mondon, M.; Fontelle N.; Désiré, J.; Lecornué, F.; Guillard, J.; Marrot, J.; Blériot, Y. Org. Lett. 2012, 14, 870. http://dx.doi.org/10.1021/ol203385w PMid:22263550

24. Takahata H. J. Pharm. Soc. Japan 2013, 133, 575.

25. Poupon, E.; Luong, B. X.; Chiaroni, A.; Kunesch, N.; Husson, H.-P. J. Org. Chem. 2000, 65, 7208. http://dx.doi.org/10.1021/jo000434c

26. Tite, T.; Tsimilaza, A.; Lallemand, M.-C.; Tillequin, F.; Leproux, P.; Libot, F.; Husson, H.-P. Eur. J. Org. Chem. 2006, 4, 863. http://dx.doi.org/10.1002/ejoc.200500754

27. Tsimilaza, A.; Tite, T.; Boutefnouchet S.; Lallemand M.-C.; Tillequin F.; Husson H.-P. Tetrahedron : Asymmetry 2007, 18, 1585. http://dx.doi.org/10.1016/j.tetasy.2007.06.015

28. Bonin, M.; Grierson, D. S.; Royer, J.; Husson, H.-P. Org. Syntheses 1992, 70, 54.

29. Guerrier, L.; Royer, J.; Grierson, D.S.; Husson, H.-P. J. Am. Chem. Soc. 1983, 105, 7754. http://dx.doi.org/10.1021/ja00364a053

30. Shing, T. K. M.; Leung, G. Y. C.; Yeung, K. W. Tetrahedron Lett. 2003, 44, 9225. http://dx.doi.org/10.1016/j.tetlet.2003.09.226

31. Shing, T. K. M.; Luk, T.; Lee, C. M. Tetrahedron 2006, 62, 6621. http://dx.doi.org/10.1016/j.tet.2006.01.116

32. Shing, T. K. M.; Luk, T. Tetrahedron : Asymmetry 2009, 20, 883. http://dx.doi.org/10.1016/j.tetasy.2009.02.039

33. Montchamp, J.-L.; Tian, F.; Hart, M. E.; Frost, J. W. J. Org. Chem. 1996, 61, 3897. http://dx.doi.org/10.1021/jo960170n

34. Grubb, L. M.; Dowdy, A. L.; Blanchette, H. S.; Friestad, G. K.; Branchaud, B. P. Tetrahedron Lett. 1999, 40, 2691. http://dx.doi.org/10.1016/S0040-4039(99)00334-2

35. Shih, T. L.; Wu S. H. Tetrahedron Lett. 2000, 41, 2957. http://dx.doi.org/10.1016/S0040-4039(00)00280-X 
36. Dinh, T. N.; Chen, A.; Chai, C. L. L. Tetrahedron 2011, 67, 3363.

http://dx.doi.org/10.1016/i.tet.2011.03.063

37. Ley, S. V.; Polara, A. J. Org. Chem. 2007, 72, 5943.

http://dx.doi.org/10.1021/jo0703451

38. Grierson, D. S.; Urrea, M.; Husson, H.-P. Heterocycles 1985, 23, 2493.

http://dx.doi.org/10.3987/R-1985-10-2493 